\title{
LIMIT SETS OF RELATIVELY HYPERBOLIC GROUPS
}

\author{
WEN-YUAN YANG
}

\begin{abstract}
In this paper, we prove a limit set intersection theorem in relatively hyperbolic groups. Our approach is based on a study of dynamical quasiconvexity of relatively quasiconvex subgroups. Using dynamical quasiconvexity, many well-known results on limit sets of geometrically finite Kleinian groups are derived in general convergence groups. We also establish dynamical quasiconvexity of undistorted subgroups in finitely generated groups with nontrivial Floyd boundary.
\end{abstract}

\section{INTRODUCTION}

The purpose of this paper is to study applications of dynamical quasiconvexity to limit sets of relatively quasiconvex subgroups. The notion of dynamical quasiconvexity is introduced by Bowditch in [5] and used to characterize a geometric notion of quasiconvexity in word hyperbolic groups. In relatively hyperbolic groups, Gerasimov-Potyagailo [13. recently showed that dynamically quasiconvex subgroups are exactly the class of relatively quasiconvex subgroups.

In this paper, we shall show that even in general convergence groups, dynamical quasiconvexity efficiently captures algebraic and geometric properties of subgroups as well. However, the particular interest we have in mind lies in relatively hyperbolic groups, and finitely generated groups with nontrivial Floyd boundary, which is conjectured to be relatively hyperbolic [22].

Let $G$ be a finitely generated group, admitting a convergence group action on a compact metric space $M$. Then the limit set $\Lambda(H)$ of a subgroup $H \subset G$ is the set of accumulation points of $H$-orbits in $M$. See Section 2 for their precise definitions. Following Anderson [3], a limit set intersection theorem for convergence groups describes the limit set $\Lambda(H \cap J)$ in terms of $\Lambda(H)$ and $\Lambda(J)$, where $H, J$ are subgroups of a convergence group $G$. Ideally, we expect such a theorem has the following form

$$
\Lambda(H) \cap \Lambda(J)=\Lambda(H \cap J) \cup E
$$

where $E$ is an exceptional set consisting of specific parabolic points of $\Lambda(H)$ and $\Lambda(J)$.

Such a limit set intersection theorem has been investigated in several different classes of groups. In 1992, Susskind-Swarup 24] showed that the above decomposition of limit sets holds for a pair of geometrically finite Kleinian subgroups. In [1,

2000 Mathematics Subject Classification. Primary 20F65, 20 F67.

Key words and phrases. dynamical quasiconvexity, relative hyperbolicity, limit sets, Floyd boundary, undistorted subgroups.

The author is supported by the China-funded Postgraduates Studying Aboard Program for Building Top University. This research was supported by National Natural Science Foundational of China (No. 11071059). 
2] and [3], using techniques specific to 3 manifolds, Anderson carried out a systematic study of the intersection of two finitely generated subgroups of 3 dimensional Kleinian groups and proved that the limit set intersection theorem holds in this context.

In 1987, Gromov [15 introduced relatively hyperbolic groups as a generalization of many naturally occurred groups, for example, word hyperbolic groups and geometrically finite Kleinian groups and many others. In word hyperbolic groups, the limit set intersection theorem is explained in Gromov [16, Page 164], where the exceptional set $E$ is empty. Our main result is to generalize these limit set intersection theorems in relatively hyperbolic groups as follow.

Theorem 1.1. Let $H, J$ be two relatively quasiconvex subgroups of a relatively hyperbolic group $G$. Then

$$
\Lambda(H) \cap \Lambda(J)=\Lambda(H \cap J) \sqcup E
$$

where the exceptional set $E$ consists of parabolic fixed points of $\Lambda(H)$ and $\Lambda(J)$, whose stabilizer subgroups in $H$ and $J$ have finite intersection. Equivalently, the set $E$ consists of the limit points isolated in $\Lambda(H) \cap \Lambda(J)$.

Remark 1.2. Two special cases of Theorem 1.1 were known in Dahmani [7. The first one is under the assumption that maximal parabolic groups are abelian. The other one is proved for a pair of fully quasiconvex subgroups, where the exceptional set $E$ is empty. By a result of Hruska [17, fully quasiconvex subgroups are relatively quasiconvex .

One corollary to Theorem 1.1 is the following well-known result, which is usually proved via geometrical methods by Hruska [17] and, independently, MartinezPedroza [21].

Corollary 1.3. Let $H, J$ be two relatively quasiconvex subgroups of a relatively hyperbolic group $G$. Then $H \cap J$ is relatively quasiconvex.

The proof of Theorem 1.1 replies crucially on dynamical quasiconvexity of relatively quasiconvex subgroups. Even in general convergence groups, dynamical quasiconvex subgroups share many nice properties with relatively quasiconvex subgroups. See Section 2 for a few of them.

The following result extends a property of quasiconvex subgroups in word hyperbolic groups proved in Mihalik-Towle [20] to dynamically quasiconvex subgroups in general convergence groups.

Theorem 1.4. Let $H$ be dynamically quasiconvex in a convergence group $G$ such that $|\Lambda(H)| \geq 2$. Then for any $g \in G \backslash H, g H g^{-1} \subseteq H$ implies that $g H^{-1}=H$.

In the final section, we explore dynamical quasiconvexity of subgroups in a special class of convergence groups, i.e. finitely generated groups with nontrivial Floyd boundary. In [10, Floyd boundary was introduced by Floyd to compactify Cayley graphs of finitely generated groups. Later, Karlsson [19] proved that the left multiplication of group elements extends to a convergence group action on Floyd boundary.

Our last result establishes the dynamical quasiconvexity of undistorted subgroups with respect to the convergence action on Floyd boundary.

Theorem 1.5. If $H$ is an undistorted subgroup of a finitely generated group $G$ with nontrivial Floyd boundary, then $H$ is dynamical quasiconvex. 
Remark 1.6. The class of groups with nontrivial Floyd boundary includes nonelementary finitely-generated relatively hyperbolic groups [12]. In particular, infiniteended groups have nontrivial Floyd boundary.

By the Floyd map theorem in 12, there exists an equivariant map from the Floyd boundary of a relatively hyperbolic group to its Bowditch boundary. Then it is easily seen that the dynamical quasiconvexity of a subgroup is kept under an equivalent quotient. See a proof in [27, Lemma 4.5], for example. This together with Theorem [1.5] gives the following result, which is first proved in [17.

Corollary 1.7. Let $H$ be an undistorted subgroup of a relatively hyperbolic group $G$. Then $H$ is relatively quasiconvex.

The paper is organized as follows. In Section 2, we define dynamical quasiconvex subgroups in general convergence groups. Then we deduce several consequences of dynamically quasiconvex subgroups in general convergence groups. In Section 3, we study the intersection of conical limit points and bounded parabolic points of dynamically quasiconvex subgroups, and then conclude with the proof of Theorem 1.1. In Section 4, it is shown that a nonparabolic dynamically quasiconvex subgroup cannot contain a proper conjugate of itself. In the final section, we briefly introduce the Floyd boundary for a finitely generated groups and give a proof of Theorem 1.5. Moreover, an example is given of dynamically quasiconvex subgroups which are not geometrically finite.

Acknowledgment. The author would like to sincerely thank Prof. Leonid Potyagailo for many helpful comments and inspired discussions during the course of this work. The author also thanks Chris Hruska for pointing out several inaccuracies of references. The author is grateful for many valuable comments of the referee, which substantially improves the presentation of the paper.

\section{Preliminary Results}

Throughout the paper, we consider a finitely generated group $G$, and a compact metrizable space $M$ containing at least three points.

A convergence group action is an action of a group $G$ on $M$ such that the induced action of $G$ on the space $\Theta M$ of distinct unordered triples of points of $M$ is properly discontinuous.

Suppose $G$ has a convergence group action on $M$. Then $M$ is partitioned into a limit set $\Lambda(G)$ and discontinuous domain $M \backslash \Lambda(G)$. The limit set $\Lambda(H)$ of a subgroup $H \subset G$ is the set of limit points, where a limit point is an accumulation point of some $H$-orbit in $M$. It is well-known that if $|\Lambda(H)| \geq 2$, the limit set $\Lambda(H)$ is also characterized as the minimal $H$-invariant closed subset in $M$ of cardinality at least two.

An element $g \in G$ is elliptic if it has finite order. An element $g \in G$ is parabolic if it has infinite order and fixes exactly one point of $M$. An element $g \in G$ is loxodromic if it has infinite order and fixes exactly two points of $M$. An infinite subgroup $P \subset G$ is a parabolic subgroup if it contains no loxodromic element. A parabolic subgroup $P$ has a unique fixed point in $M$. This point is called a parabolic point. The stabilizer of a parabolic point is always a maximal parabolic group. A parabolic point $p$ with stabilizer $G_{p}:=\operatorname{Stab}_{G}(p)$ is bounded if $G_{p}$ acts cocompactly on $M \backslash\{p\}$. A point $z \in M$ is a conical limit point if there exists a sequence $\left\{g_{i}\right\}$ 
in $G$ and distinct points $a, b \in M$ such that $g_{i}(z) \rightarrow a$, while for all $q \in M \backslash\{z\}$ we have $g_{i}(q) \rightarrow b$.

Before discussing relative hyperbolicity, we recall the following well-known result on general convergence groups.

Lemma 2.1. 25], Theorem 3.A] In a convergence group, a conical limit point can not be parabolic.

In the literature, various definitions of relative hyperbolicity were proposed, see Farb 9, Bowditch 4, Osin 23, Drutu-Sapir 6] and so on. These different definitions are now proven to be equivalent for countable groups, see Hruska 17 for a complete account, and provide convenient and complement viewpoints to the study of this class of groups. For the sake of the purpose of this paper, we use the following dynamical formulation of relatively hyperbolic groups.

Definition 2.2. A convergence group action of $G$ on $M$ is geometrically finite if every limit point of $G$ is either conical or bounded parabolic. Let $\mathbb{P}$ be a set of representatives of the conjugacy classes of maximal parabolic subgroups. Then we say the pair $(G, \mathbb{P})$ is relatively hyperbolic. When $\mathbb{P}$ is clear in the context, we just say $G$ is relatively hyperbolic.

Remark 2.3. By the work of Drutu-Sapir [6], Osin [23] and Gerasimov [11, maximal parabolic subgroups are quasiconvex and finitely generated. So in the definition of relative hyperbolicity, we do not need impose the "finitely generated" condition on maximal parabolic subgroups, as usually do in Bowditch [4].

The following notion of dynamical convexity is introduced by Bowditch [5] and proven to be equivalent to the geometric quasiconvexity in word hyperbolic groups.

Definition 2.4. A subgroup $H$ of a convergence group $G$ is dynamically quasiconvex if the following set

$$
\{g H \in G / H: g \Lambda(H) \cap K \neq \emptyset \text { and } g \Lambda(H) \cap L \neq \emptyset\}
$$

is finite, whenever $K$ and $L$ are disjoint closed subsets of $M$.

Recently, Gerasimov-Potyagailo [13] proved that dynamical quasiconvexity coincides with relatively quasiconvexity in relatively hyperbolic groups, which gives a positive answer to a question of Osin in his book [23]. We refer the reader to [23] for the definition of relative quasiconvexity.

Theorem 2.5. [13] Suppose $G$ is relatively hyperbolic. Every subgroup $H$ of $G$ is dynamically quasiconvex if and only if it is relatively quasiconvex.

Let us first draw some consequences of dynamical quasiconvexity, without assuming $G$ is relatively hyperbolic.

Lemma 2.6. Let $H$ be dynamically quasiconvex in a convergence group $G$ such that $|\Lambda(H)| \geq 2$. Then for any subgroup $H \subset J \subset G$ satisfying $\Lambda(H)=\Lambda(J)$, we have that $H$ is of finite index in $J$. In particular, $J$ is dynamically quasiconvex.

Proof. Since $|\Lambda(H)| \geq 2$, we can pick distinct points $x$ and $y$ from $\Lambda(H)$. Since $\Lambda(H)=\Lambda(J)$, we have that each coset of $H$ in $J$ belongs to the following set

$$
\{g H \in G / H: g \Lambda(H) \cap\{x\} \neq \emptyset \text { and } g \Lambda(H) \cap\{y\} \neq \emptyset\} .
$$


By the dynamical quasiconvexity of $H$, the above set is finite. Thus, $H$ is of finite index in $J$.

In order to prove the dynamical quasiconvexity of $J$, it suffices to show that the following set

$$
\Omega:=\{g J \in G / J: g \Lambda(J) \cap K \neq \emptyset \text { and } g \Lambda(J) \cap L \neq \emptyset\}
$$

is finite, for any given disjoint closed subsets $K, L \subset M$. On the other hand, since $H$ is dynamically quasiconvex, the following set

$$
\{g H \in G / H: g \Lambda(H) \cap K \neq \emptyset \text { and } g \Lambda(H) \cap L \neq \emptyset\} .
$$

is finite. Combining with the fact $H$ is of finite index in $J$, we have that the above set $\Omega$ is finite. Therefore, $J$ is dynamically quasiconvex.

Corollary 2.7. Let $H, J$ be dynamically quasiconvex in a convergence group $G$ such that $\Lambda(H)=\Lambda(J)$. If $|\Lambda(H)| \geq 2$, then $H$ and $J$ are commensurable.

Proof. Let $L$ be the stabilizer in $G$ of the limit set $\Lambda(H)$. Using Lemma 2.6, we have that $H, J$ are both of finite index in $L$. It thus follows that $H \cap J$ is of finite index in both $H$ and $J$.

Recall that the commensurator of $H$ in a convergence group $G$ is defined as the subgroup of $G$, consisting of all $g \in G$ such that $H \cap g \mathrm{Hg}^{-1}$ has finite index in both $H$ and $g H g^{-1}$.

Corollary 2.8. Let $H$ be dynamically quasiconvex in a convergence group $G$ such that $|\Lambda(H)| \geq 2$. Then $H$ is of finite index in its commensurator. In particular, $H$ is of finite index in its normalizer.

Proof. Let $L$ be the commensurator of $H$ in $G$. Then $H \subset L$. It is obvious that $\Lambda(H) \subset \Lambda(L)$.

It is well-known that the limit set of a subgroup is same as the one of its finite extension. So for each $g \in L$, we have $\Lambda(H)=\Lambda\left(g H g^{-1}\right)=g \Lambda(H)$, i.e. $L$ leaves invariant the limit set $\Lambda(H)$. Since the limit set $\Lambda(L)$ is the minimal $L$ invariant closed subset in $M$ of cardinality at least two, we have $\Lambda(L)=\Lambda(H)$. The conclusion now follows from Lemma 2.6

Remark 2.9. In relatively hyperbolic groups, Corollary 2.8 has been proven using different methods in Hruska-Wise [18. We remark the hypothesis $|\Lambda(H)| \geq 2$ is necessary for the above lemma and corollaries, as it is easy to get counterexamples when $H$ is taken as a parabolic subgroup.

\section{INTERSECTIONS OF LIMIT SETS}

In this section, we study the intersection of limit sets of dynamically quasiconvex subgroups. The intersection of conical limit points is firstly examined.

Proposition 3.1. Let $H$ be dynamically quasiconvex in a convergence group $G$. Suppose $J<G$ is infinite and let $z \in \Lambda(H) \cap \Lambda(J)$ be a conical limit point of $J$. Then $z \in \Lambda(H \cap J)$ is a conical limit point of $H \cap J$.

Proof. Let $z \in \Lambda(H) \cap \Lambda(J)$ be a conical limit point of $J$. Then there exists a sequence $\left\{j_{n}\right\}$ in $J$ and distinct points $a, b \in \Lambda(J)$ such that $j_{n}(z) \rightarrow a$, while $j_{n}(q) \rightarrow b$ for all $q \in \Lambda(J) \backslash\{z\}$. By the convergence property of $\left\{j_{n}\right\}$, we also have that $j_{n}(q) \rightarrow b$ for all $q \in M \backslash\{z\}$. In particular, we can choose $q$ to be a limit 
point in $\Lambda(H) \backslash\{z\}$. Here, we use the fact $|\Lambda(H)| \geq 2$, which follows from Lemma 2.1 .

Take closed neighborhoods $U$ and $V$ of $a$ and $b$ respectively, such that $U \cap V=\emptyset$. After passage to a subsequence of $\left\{j_{n}\right\}$, we can assume $j_{n}(z) \in U$ and $j_{n}(q) \in V$ for all $n$. This implies that $j_{n} H$ belongs to the following set for all $n$,

$$
\{g H \in G / H: g \Lambda(H) \cap U \neq \emptyset \text { and } g \Lambda(H) \cap V \neq \emptyset\} .
$$

By the dynamical quasiconvexity of $H$ in $G$, the above set is finite. Thus, $\left\{j_{n} H\right\}$ is a finite set of cosets. By taking further a subsequence of $\left\{j_{n}\right\}$, we suppose $j_{n} H=j_{1} H$ for all $n$. We can write $j_{n}=j_{1} h_{n}$ for each $n$, where $h_{n} \in H$. Then $j_{1}^{-1} j_{n}=h_{n}$ implies that $H \cap J$ is nontrivial and infinite.

It suffices to prove that $z$ is a conical limit point of $H \cap J$. By the convergence property of $\left\{j_{n}\right\}$, it follows that $h_{n}(z)=j_{1}^{-1} j_{n}(z) \rightarrow j_{1}^{-1}(a)$ and $h_{n}(q)=$ $j_{1}^{-1} j_{n}(q) \rightarrow j_{1}^{-1}(b)$ for all $q \in M \backslash\{z\}$. Thus, $z$ is a conical limit point of $H \cap J$.

Remark 3.2. A similar statement of Proposition 3.1]in relatively hyperbolic groups appears in the proof of Proposition 3.1.10 in [7].

We now study how bounded parabolic points intersect. Compared to that of conical points, the intersection of bounded parabolic points raises some complicated behavior.

Proposition 3.3. Let $H, J$ be infinite subgroups of a convergence group $G$. If $z \in \Lambda(H) \cap \Lambda(J)$ is a bounded parabolic point of $H$ and $J$, then $z$ is either a bounded parabolic point of $H \cap J$, or an isolated point in $\Lambda(H) \cap \Lambda(J)$ and does not lie in $\Lambda(H \cap J)$.

Proof. Since $z$ is a bounded parabolic point of both $H$ and $J$, there are compact subsets $K \subset \Lambda(H) \backslash z$ and $L \subset \Lambda(J) \backslash\{z\}$, such that $H_{z} K=\Lambda(H) \backslash\{z\}$ and $J_{z} L=\Lambda(J) \backslash\{z\}$. Here, $H_{z}$ and $J_{z}$ are stabilizers in $H$ and $J$ of $z$, respectively. Let $P=H_{z} \cap J_{z}$.

We claim that there exists a compact subset $C \subset M \backslash\{z\}$ such that $\Lambda(H \cap J) \backslash z \subset$ $P C$.

Note first that $\Lambda(H \cap J) \backslash\{z\} \subset(\Lambda(H) \cap \Lambda(J)) \backslash z=H_{z} K \cap J_{z} L$. Therefore, it suffices to show that there exists a compact subset $C \subset M \backslash\{z\}$ such that $H_{z} K \cap J_{z} L \subset P C$. Since $G$ is countable, we define the following set

$$
\mathcal{A}=\left\{h_{n} K \cap j_{n} L:\left(h_{n}, j_{n}\right) \in H_{z} \times J_{z}, h_{n} K \cap j_{n} L \neq \emptyset\right\} .
$$

We remark that it is possible that one set $h K$ may have nontrivial intersections with two more sets $j_{1} L$ and $j_{2} L$, but $\left(h, j_{1}\right)$ and $\left(h, j_{2}\right)$ are counted differently in the set $\mathcal{A}$. Note that $H_{z} K \cap J_{z} L \subset \cup \mathcal{A}$.

Define the set $\mathcal{B}=\left\{j_{n}^{-1} h_{n}: j_{n}^{-1} h_{n} K \cap L \neq \emptyset,\left(h_{n}, j_{n}\right) \in H_{z} \times J_{z}\right\}$. We now show that $\mathcal{B}$ is finite. Suppose not. By the convergence property, there exists an infinite subsequence $\left\{j_{n_{i}}^{-1} h_{n_{i}}\right\}$ of $\mathcal{B}$ such that $\left\{j_{n_{i}}^{-1} h_{n_{i}}\right\}$ converges locally compactly to $b$ on $M \backslash\{a\}$, for some $a, b \in M$. We claim $a=b$. Otherwise, using Lemma 2.5 in Bowditch [5], we have that $j_{n_{i}}^{-1} h_{n_{i}}$ are loxodromic elements for all sufficiently large $n_{i}$. But this contradicts to the fact that $\left\{j_{n_{i}}^{-1} h_{n_{i}}\right\}$ lie in the maximal parabolic subgroup $G_{z}$.

Moreover, we have that $a=b=z$, since $z$ is the fixed point of elements $j_{n}^{-1} h_{n}$. Note that $K \subset M \backslash\{z\}$ and $L \subset M \backslash\{z\}$ are disjoint compact subsets. Since 
$j_{n_{i}}^{-1} h_{n_{i}} K \cap L \neq \emptyset$, the subsequence $\left\{j_{n_{i}}^{-1} h_{n_{i}}\right\}$ is a finite set by the convergence property. This is a contradiction. Hence $\mathcal{B}$ is a finite set.

Let $\mathcal{B}$ be a finite set, say $\left\{j_{1}^{-1} h_{1}, \ldots, j_{r}^{-1} h_{r}\right\}$, for example. Without loss of generality, we first consider the elements in $\left\{j_{n}^{-1} h_{n}\right\}$ of the form $j_{n}^{-1} h_{n}=j_{1}^{-1} h_{1}$. Then $j_{n} j_{1}^{-1}=h_{n} h_{1}^{-1} \in H_{z} \cap J_{z}=P$. We write $j_{n}=p_{n} j_{1}$ and $h_{n}=p_{n} h_{1}$ for some $p_{n} \in P$. So we have $h_{n} K \cap j_{n} L=p_{n}\left(h_{1} K \cap j_{1} L\right)$ for each $j_{n}^{-1} h_{n}=j_{1}^{-1} h_{1}$.

We can do the rewriting process similarly for other elements in $\left\{j_{n}^{-1} h_{n}\right\}$, and finally we obtain $H_{z} K \cap J_{z} L \subset \cup \mathcal{A} \subset P C$, where $C$ is a compact set defined as $\bigcup_{i=1}^{r}\left(h_{i} K \cap j_{i} L\right)$. The claim is proved.

Recall that we have proved there exists a compact subset $C \subset M$ such that the following holds

$$
\Lambda(H \cap J) \backslash\{z\} \subset(\Lambda(H) \cap \Lambda(J)) \backslash\{z\} \subset P C .
$$

We now have two cases to consider for finishing the proof of proposition,

$P$ is finite. Since the right-hand of (2) is a compact set, there exists an open neighborhood of $z$ disjoint with $\Lambda(H) \cap \Lambda(J)$. Thus, $z$ is an isolated point of $\Lambda(H) \cap \Lambda(J)$ and does not lie in $\Lambda(H \cap J)$.

$P$ is infinite. $P$ acts cocompactly on $\Lambda(H \cap J) \backslash\{z\}$. Thus, $z$ is a bounded parabolic point of $H \cap J$.

Summarizing the above results, we can now conclude with the proof of Theorem 1.1. Recall that by Theorem 2.5, dynamically quasiconvex subgroups coincide with relatively quasiconvex groups in relatively hyperbolic groups.

Proof of Theorem 1.1. By (QC-1) definition of relative quasiconvexity in [17, a relatively quasiconvex subgroup acts on its limit set as a geometrically finite convergence action. Then the limit set of a relatively quasiconvex subgroup consists of conical limit points and bounded parabolic points. Therefore, the decomposition of $\Lambda(H) \cap \Lambda(J)$ follows from Propositions 3.1 and 3.3 .

Remark 3.4. In word hyperbolic groups, the exceptional set $E$ is empty since there are no parabolic subgroups. In this case, limit sets of two relatively quasiconvex subgroups intersect at least in two points, once they intersect. But in the relative case, it is possible that their limit sets intersect in only one (necessarily parabolic) point. For example, let $H=\langle z+1\rangle, J=\langle z+i\rangle$ be two parabolic subgroups of a Fuchsian group $G$ acting on the upper half space $\{z \in \mathbb{C}: \Im(z)>0\}$. Note that the intersection $H \cap J$ is trivial, but $H$ and $J$ share the same fixed point $\infty$.

The following corollary follows from the isolatedness of the exceptional set $E$.

Corollary 3.5. Let $H, J$ be two relatively quasiconvex subgroups of $G$. If $\Lambda(H) \subset$ $\Lambda(J)$. Then either $\Lambda(H \cap J)=\Lambda(H)$ or $H$ is a parabolic subgroup.

Proof. Suppose $H$ is not a parabolic subgroup. Then $|\Lambda(H)| \geq 2$. By Theorem 1.1. we have $\Lambda(H)=\Lambda(H \cap J) \sqcup E$, where $E$ consists of isolated points in $\Lambda(H)$. It is well-known that limit sets are perfect, if containing at least 3 points. So if $|\Lambda(H)|>2$, then $E$ is empty.

It suffices to consider the case $|\Lambda(H)|=2$. In this case, $H$ is a virtually cyclic group. Thus, $\Lambda(H)$ consists of two conical limit points. By Lemma 2.1, we have that $E$ is empty. 


\section{Proper Conjugates of Dynamically Quasiconvex Subgroups}

Suppose $G$ has a convergence group action on $M$. According to [14, a subgroup $H \subset G$ is said to be maximal in its limit set if $H=\operatorname{Stab}_{G}(\Lambda(H))$. Recall that Lemma 2.6 shows any nonparabolic dynamically quasiconvex subgroup is of finite index in the stabilizer of its limit set.

Lemma 4.1. Let $H$ be dynamically quasiconvex in a convergence group $G$ and suppose $H$ is maximal in its limit set. Then for any $g \in G \backslash H, g \Lambda(H) \nsubseteq \Lambda(H)$.

Proof. If $|\Lambda(H)|=1$, then $H$ is the maximal parabolic subgroup. In this case, the conclusion is trivial. We now consider the case $|\Lambda(H)| \geq 2$. By way of contradiction, we suppose that $g \Lambda(H) \subseteq \Lambda(H)$.

Take distinct points $x$ and $y$ from $\Lambda(H)$. Since $g \Lambda(H) \subseteq \Lambda(H)$, we have $g^{n}(x) \in$ $\Lambda(H)$ and $g^{n}(y) \in \Lambda(H)$ for each $n \in \mathbb{N}$. Therefore, we have that the cosets $g^{-n} H$ belong to the following set

$$
\{g H \in G / H: g \Lambda(H) \cap\{x\} \neq \emptyset \text { and } g \Lambda(H) \cap\{y\} \neq \emptyset\} .
$$

Since $H$ is dynamically quasiconvex, we have the set $\left\{g^{-n} H\right\}$ is finite. Consequently there exist two different integers $m$ and $n$ such that $g^{-m} H=g^{-n} H$, and thus $g^{n-m} \in H$. Then $\Lambda(H)=g^{n-m} \Lambda(H) \subseteq g \Lambda(H) \subseteq \Lambda(H)$. Hence $\Lambda(H)=g \Lambda(H)$, which is impossible since $H$ is maximal in its limit set $\Lambda(H)$.

Remark 4.2. Lemma 4.1]generalizes Lemma 2.10 in Gitik-Mitra-Rips-Sageev [14].

We now prove Theorem 1.4 .

Proof of Theorem 1.4. Let $g$ be an element of $G \backslash H$ such that $g H g^{-1} \subseteq H$. By Lemma 4.1, it follows that $g$ belongs to the setwise stabilizer $K$ in $G$ of $\Lambda(H)$. Using Lemma 2.6, we obtain that $H$ is of finite index in $K$. So $g^{n}$ belongs to $H$ for some $n$. Thus, we have $H=g^{n} H g^{-n} \subset g H g^{-1} \subset H$. The proof is complete.

Remark 4.3. The condition that $|\Lambda(H)| \geq 2$ could not be dropped. It is known that there exists a finitely generated group $G$ containing a finitely generated subgroup $H$ such that, for some $g \in G, g H g^{-1} \subset H$ but $g H^{-1} \subsetneq H$. See [26] for an elementary example. We then form a free product $G * F_{2}$, where $F_{2}$ is a free group of rank 2 . By the second definition of relative hyperbolicity in [4, $G * F_{2}$ is relatively hyperbolic. In particular, $G$ is a maximal parabolic subgroup. But $H \subset G * F_{2}$ doesnot satisfy the statement of Theorem 1.4 for some $g \in G$.

\section{Undistorted Subgroups of Groups with Nontrivial Floyd BOUNDARY}

In this section, we consider a finitely generated group $G$ with a fixed finite generating set $S$, without assuming relative hyperbolicity of $G$.

As usual, $S$ is assumed to be symmetric, i.e. $S=S^{-1}$. Then the Cayley graph $\Gamma(G, S)$ of $G$ with respect to $S$, is defined as an oriented graph with vertex set $G$ and edge set $G \times S$. An edge $(g, s) \in G \times S$ goes from $g$ to $g s$. Note that $\Gamma(G, S)$ is a connected graph, which induces a word metric $d_{S}$ on $G$ by setting the length of each edge to be 1 .

Given a rectifiable path $p$ in $\Gamma(G, S)$, we denote by $p_{-}, p_{+}$the initial and terminal endpoint of $p$ respectively. Let $l(p)$ be the length of $p$. We say $p$ is a $\epsilon$-quasigeodesic 
for a constant $\epsilon \geq 0$ if, for any subpath $q$ of $p$, we have $l(q)<\epsilon d_{S}\left(q_{-}, q_{+}\right)+\epsilon$. Let $d_{S}(1, p)$ be the distance from the identity to the path $p$ with respect to $d_{S}$.

Recall that a $(\epsilon-)$ quasi-isometric map $\phi: X \rightarrow Y$ between two metric spaces $\left(X, d_{X}\right)$ and $\left(Y, d_{Y}\right)$ is a map such that the following holds

$$
\epsilon^{-1} d_{X}(x, y)-\epsilon \leq d_{Y}(\phi(x), \phi(y)) \leq \epsilon d_{X}(x, y)+\epsilon .
$$

Definition 5.1. Let $H \subset G$ be a finitely generated subgroup with a finite generating set $T$. Then $H$ is undistorted if the inclusion of $\left(H, d_{T}\right)$ into $\left(G, d_{S}\right)$ is a quasi-isometric map.

Note that the definition of an undistorted subgroup is independent of choices of finite generating sets $S, T$. Without loss of generality, we assume that $T \subset S$ in the sequel. Then the embedding $\imath: \Gamma(H, T) \hookrightarrow \Gamma(G, S)$ is a quasi-isometric map. In particular, a geodesic in $\Gamma(H, T)$ is naturally embedded as a quasigeodesic in $\Gamma(G, S)$.

We now briefly discuss the construction of Floyd boundary of a finitely generated group. We refer the reader to [10, [19] and [13] for more details.

Let $f: \mathbb{N} \rightarrow \mathbb{R}$ be the function $f(n)=n^{-2}$. We rescale the length of each edge $e$ of $\Gamma(G, S)$ by a factor $f\left(d_{S}(1, e)\right)$, and then take the Cauchy metric completion $\bar{G}$. Denote by $\rho$ the complete metric on $\bar{G}$. Then Floyd boundary $\partial(G)$ is defined as $\bar{G} \backslash G$. With a change of finite generating sets, Floyd boundary is well-defined up to a bi-Lipschitz homeomorphism.

If $\partial(G)$ consists of 0,1 or 2 points then it is said to be trivial. Otherwise, it is uncountable and is called nontrivial. The class of groups with nontrivial Floyd boundary includes non-elementary relatively hyperbolic groups 12 .

In [19, Karlsson showed that if Floyd boundary is nontrivial, then $G$ acts on $\partial(G)$ as a convergence group action. In what follows, when speaking of limit sets and dynamical quasiconvexity of subgroups in $G$, we have in mind the convergence action of $G$ on $\partial(G)$.

The following lemma shows that the Floyd length of a far (quasi)geodesic in $\Gamma(G, S)$ is small. The original version was stated in [19] for geodesics, but its proof also works for quasigeodesic in the Cayley graph.

Lemma 5.2. [19] Given $\epsilon>0$, there is a function $\Theta_{\epsilon}: \mathbb{N} \rightarrow \mathbb{R}_{\geq 0}$ such that $\Theta_{\epsilon}(n) \rightarrow$ 0 as $n \rightarrow \infty$ and the following property holds. Let $z, w$ be two points in $G$ and let $\gamma$ be an $\epsilon$-quasigeodesic between $z$ and $w$ of $\Gamma(G, S)$. Then the following holds

$$
\rho(z, w) \leq \Theta_{\epsilon}\left(d_{S}(1, \gamma)\right)
$$

Recall that we assume $T \subset S$ and the embedding $\imath: \Gamma(H, T) \hookrightarrow \Gamma(G, S)$ is quasi-isometric. The following lemma roughly says that any two limit points of an undistorted subgroup $H$ can be connected by a geodesic in $\Gamma(H, T)$.

Lemma 5.3. If $H$ is undistorted in $G$ such that $\Lambda(H) \geq 2$, then there exists a constant $\epsilon_{0} \geq 0$ such that the following holds. For any two distinct points $p, q \in$ $\Lambda(H)$, there exists an $\epsilon_{0}$-quasigeodesic $\gamma$ in $\Gamma(G, S)$ between $p$ and $q$ such that $\gamma \subset \Gamma(H, T)$.

Proof. Since $p$ and $q$ are distinct limit points of $H$, there exist two sequences $\left\{h_{n}\right\}$ and $\left\{h_{n}^{\prime}\right\}$ of $H$ such that $h_{n} \rightarrow p$ and $h_{n}^{\prime} \rightarrow q$. Let $\delta=\rho(p, q) / 3$.

Without loss of generality, we assume for all $n, h_{n} \in B_{\delta}(p)$ and $h_{n}^{\prime} \in B_{\delta}(q)$, after passage to subsequences of $\left\{h_{n}\right\}$ and $\left\{h_{n}^{\prime}\right\}$ respectively. Here, $B_{\delta}(p)$ and 
$B_{\delta}(q)$ denote open metric balls centered at $p$ and $q$ in $\bar{G}$ with radius $\delta$ respectively. It then follows by the triangle inequality that $\rho\left(h_{n}, h_{n}^{\prime}\right)>d(p, q) / 3$ for all $n$.

Taking geodesics $\gamma_{n}$ in the Cayley graph $\Gamma(H, T)$ such that $\left(\gamma_{n}\right)_{-}=h_{n}$ and $\left(\gamma_{n}\right)_{+}=h_{n}^{\prime}$. By the undistortedness of $H$, there is a positive constant $\epsilon_{0}$ depending on $H$, such that any geodesic in $\Gamma(H, T)$ is an $\epsilon_{0}$-quasigeodesic in $\Gamma(G, S)$. Thus, $\gamma_{n}$ are $\epsilon_{0}$-quasigeodesics in $\Gamma(G, S)$. Observe that the endpoints $h_{n}, h_{n}^{\prime}$ of $\gamma_{n}$ have at least a $\rho$-distance $\delta$ in $\bar{G}$.

Let $\Theta_{\epsilon_{0}}$ be the function given by Lemma 5.2. Since $\Theta_{\epsilon_{0}}(n) \rightarrow 0$ as $n \rightarrow \infty$, let $R$ be the maximal integer $m$ such that $\Theta_{\epsilon_{0}}(m) \geq \delta$. By Lemma 5.2. each quasigeodesic $\gamma_{n}$ intersects a closed ball $B$ centered at identity with radius $R$ in $\Gamma(G, S)$.

Therefore, using a Cantor diagonal argument based on $\gamma_{n}$, we obtain an $\epsilon_{0^{-}}$ quasigeodesic $\gamma$ in $\Gamma(G, S)$ between $p$ and $q$ such that the vertex set of $\gamma$ lies in $H$.

Remark 5.4. In contrast with hyperbolic groups, two (quasi)geodesics in $\Gamma(G, S)$ with same endpoints may not be uniformly Hausdorff distance bounded. Thus, we could not guarantee that any (quasi)geodesic between $p$ and $q$ satisfies the statement of Lemma 5.3 .

We are now ready to prove Theorem 1.5 .

Proof of Theorem 1.5. It suffices to establish the conclusion under the assumption $|\Lambda(H)| \geq 2$. We are going to bound the following set

$$
\{g H \in G / H: g \Lambda(H) \cap L \neq \emptyset \text { and } g \Lambda(H) \cap K \neq \emptyset\},
$$

whenever $K$ and $L$ are disjoint closed subsets of $\partial(G)$.

Suppose, to the contrary, there exists a sequence of distinct cosets $g_{n} H$ such that $g_{n} \Lambda(H) \cap K \neq \emptyset$ and $g_{n} \Lambda(H) \cap L \neq \emptyset$. Let $p_{n} \in g_{n} \Lambda(H) \cap K$ and $q_{n} \in g_{n} \Lambda(H) \cap L$. Note that $g_{n}^{-1}\left(p_{n}\right), g_{n}^{-1}\left(q_{n}\right) \in \Lambda(H)$. By Lemma [5.3. we obtain $\epsilon_{0}$-quasigeodesics $\gamma_{n}$ between $g_{n}^{-1}\left(p_{n}\right)$ and $g_{n}^{-1}\left(q_{n}\right)$, such that the vertex set of $\gamma_{n}$ lies in $H$. Hence $g_{n}\left(\gamma_{n}\right)$ are $\epsilon_{0}$-quasigeodesics with endpoints $p_{n}, q_{n} \in g_{n} \Lambda(H)$, such that the vertex set of $g_{n}\left(\gamma_{n}\right)$ lies in the same coset $g_{n} H$.

Note that $\left\{p_{n}, q_{n}\right\} \in K \times L$. Since $K \times L$ is compact in $\partial(G) \times \partial(G)$. there exists a uniform positive constant $\mu$ depending on $K$ and $L$, such that $\rho\left(p_{n}, q_{n}\right) \geq \mu$ for all $n$. Let $\Theta_{\epsilon_{0}}$ be the function given by Lemma 5.2. Since $\Theta_{\epsilon_{0}}(n) \rightarrow 0$ as $n \rightarrow \infty$, let $R$ be the maximal integer $m$ such that $\Theta_{\epsilon_{0}}(m) \geq \mu$.

By Lemma [5.2, any $\epsilon_{0}$-quasigeodesic between $p_{n}$ and $q_{n}$ intersects non-trivially with $B$, where $B$ is the closed ball at the identity with radius $R$ in $\Gamma(G, S)$. Let $c_{n}$ be an intersection point of $g_{n} \gamma_{n} \cap B$. Then we have $d_{S}\left(1, c_{n}\right)<R$ for every $n$.

Recall that the vertex set of $g_{n}\left(\gamma_{n}\right)$ lies in $g_{n} H$. Therefore, for each $n$, there exists $h_{n} \in H$ such that $d_{S}\left(g_{n} h_{n}, c_{n}\right)<1$. Then $d_{S}\left(1, g_{n} h_{n}\right)<R+1$ for all $n$. Since $S$ is a finite set, we have the set $\left\{g_{n} h_{n}\right\}$ is finite. This is a contradiction, as $\left\{g_{n} H\right\}$ is assumed as a sequence of different $H$-cosets in $G$. The proof is complete.

In view of Theorem 1.5 the previous Lemma 2.6. Corollaries 2.7, 2.8 and Theorem 1.4 can be stated in the setting of finitely generated groups with nontrivial Floyd boundary. In favor of applications in group theory, we state the following.

Corollary 5.5. Let $H$ be undistorted in $G$ such that $|\Lambda(H)| \geq 2$. Then $H$ is of finite index in its commensurator. In particular, $H$ is of finite index in its normalizer. 
Corollary 5.6. Let $H$ be undistorted in $G$ such that $|\Lambda(H)| \geq 2$. Then for any $g \in G \backslash H, g H g^{-1} \subseteq H$ implies that $g H g^{-1}=H$.

In relatively hyperbolic groups, the limit set of a relatively quasiconvex subgroup consists of conical points and bounded parabolic points. This fact allows us to complete the limit set intersection theorem 1.1 for relatively quasiconvex groups. In general convergence groups it is an interesting question to ask whether dynamically quasiconvex subgroups act geometrically finitely on their limit sets. The following example gives a negative answer to the question.

Example 5.7. Dunwoody's inaccessible group $J$ in 8 has infinite ends and thus nontrivial Floyd boundary. Since infinitely ended groups are relatively hyperbolic, by using a theorem of Stalling with the second definition of relative hyperbolicity in [4. Thus, $J$ is relatively hyperbolic. Let $\mathbb{H}$ be a set of representatives of conjugacy class of maximal parabolic subgroups of $J$. Since $(J, \mathbb{H})$ is relatively hyperbolic, then each $H \in \mathbb{H}$ is undistorted in $J$. See the remark 2.3 .

In [27, we prove that if $(G, \mathbb{P})$ is relatively hyperbolic, then $G$ acts geometrically finitely on $\partial G$ if and only if each $P \in \mathbb{P}$ acts geometrically finitely on its limit set $\Lambda(P) \subset \partial G$.

However, it is noted in 27 that $J$ doesnot act geometrically finitely on its Floyd boundary $\partial J$. Therefore, there exists at least one $H \in \mathbb{H}$ such that $H$ doesnot act geometrically finitely on its limit set $\Lambda(H) \subset \partial J$. Moreover, $\Lambda(H)$ contains infinite limit points, otherwise $J$ would act geometrically finitely on $\partial J$.

In a word, $H$ is dynamically quasiconvex by Theorem 1.5 but doesnot act geometrically finitely on its limit set in the Floyd boundary $\partial J$.

\section{REFERENCES}

1. J. Anderson, On the finitely generated intersection property for Kleinian groups. Complex Variables Theory Appl. 17 (1991), 111-112.

2. J. Anderson, Intersections of topologically tame subgroups of Kleinian groups. J. Anal. Math. 65 (1995), 77-94.

3. J. Anderson, The limit set intersection theorem for finitely generated Kleinian groups. Math. Res. Lett. 3 (1996), 675-692.

4. B. Bowditch, Relatively hyperbolic groups. Preprint, Univ. of Southampton, 1999.

5. B. Bowditch, Convergence groups and configuration spaces. in "Group Theory Down Under" (J. Cossey, C.F. Miller, W.D. Neumann, M. Shapiro, eds.), de Gruyter (1999), 23-54.

6. C. Drutu and M. Sapir. Tree-graded spaces and asymptotic cones of groups. With an appendix by D. Osin and M. Sapir. Topology, 44(5)(2005) 959-1058.

7. F. Dahmani. Combination of convergence groups. Geom. Topol., 7 (2003) 933-963.

8. M. Dunwoody. An inaccessible group. In Geometric group theory, Vol. 1 (Sussex, 1991), volume 181 of London Math. Soc. Lecture Note Ser., pages 75-78. Cambridge Univ. Press, Cambridge, 1993.

9. B. Farb, Relatively hyperbolic groups. Geom. Funct. Anal. 8(5) (1998), 810-840.

10. W. Floyd, Group completions and limit sets of Kleinian groups. Inventiones Math. 57 (1980), 205-218.

11. V. Gerasimov, Expansive convergence groups are relatively hyperbolic. Geom. Funct. Anal. 19 2009, 137-169.

12. V. Gerasimov, Floyd maps to the boundaries of relatively hyperbolic groups. preprint 2010.

13. V. Gerasimov and L. Potyagailo, Dynamical quasiconvexity in relatively hyperbolic groups. preprint 2009

14. R. Gitik, M. Mitra, E. Rips and M. Sageev, Widths of subgroups. Trans. Amer. Math. Soc. 350 (1998), 321-329

15. M. Gromov, Hyperbolic groups. from: Essays in group theory (S Gersten, editor), Springer, New York (1987),75-263. 
16. M. Gromov, Asymptotic invariants of infinite groups. In Geometric group theory, Vol. 2 (Sussex, 1991), volume 182 of London Math. Soc. Lecture Note Ser., pages 1-295. Cambridge Univ. Press, Cambridge, 1993.

17. G. Hruska, Relative hyperbolicity and relative quasiconvexity for countable groups. Algebr. Geom. Topol. 10 (2010) 1807-1856.

18. G. Hruska and D. Wise, Packing subgroups in relatively hyperbolic groups. Geom. Topol. 13(4) (2009),1945-1988.

19. A. Karlsson, Free subgroups of groups with non-trivial Floyd boundary. Comm. Algebra, 31 (2003), 5361-5376.

20. M. Mihalik, W. Towle, Quasiconvex subgroups of negatively curved groups. Pure and Applied Algebra, 95 (1994), 297-301.

21. Eduardo Martinez-Pedroza, Combination of Quasiconvex Subgroups of Relatively Hyperbolic Groups. Groups, Geometry, and Dynamics, 3 (2009), 317-342.

22. A. Olshanskii, D. Osin, M. Sapir, Lacunary hyperbolic groups. With an appendix by Michael Kapovich and Bruce Kleiner. Geom. Topol. 13 (2009), no. 4, 2051-2140.

23. D. Osin, Relatively hyperbolic groups: intrinsic geometry, algebraic properties and algorithmic problems. Mem. Amer. Math. Soc., 179(843) 2006, 1-100.

24. P. Susskind and G. Swarup, Limit sets of geometrically finite hyperbolic groups. Amer. J. Math. 114 (1992), 233-250.

25. P. Tukia, Conical limit points and uniform convergence groups. J. Reine. Angew. Math. 501 (1998) 71-98.

26. S. Wang, Q. Zhou, On the proper conjugation of kleinian groups, Geom Dedicata 56, 145154(1995).

27. Wenyuan Yang, Peripheral structures of relatively hyperbolic groups, Preprint 2010.

College of Mathematics and Econometrics, Hunan University, Changsha, Hunan 410082 People's Republic of China

Current address: U.F.R. de Mathematiques, Universite de Lille 1, 59655 Villeneuve D'Ascq Cedex, France

E-mail address: wyang@math.univ-lille1.fr 